\title{
Resting state connectivity between default mode network and insula encodes acute migraine headache
}

Cephalalgia

0(0) I-9

(C) International Headache Society 2017 Reprints and permissions: sagepub.co.uk/journalsPermissions.nav DOI: $10.1177 / 0333102417715230$ journals.sagepub.com/home/cep

(\$SAGE

\author{
Gianluca Coppola', Antonio Di Renzo', Emanuele Tinelli ${ }^{2}$, \\ Cherubino Di Lorenzo ${ }^{3}$, Marco Scapeccia ${ }^{2}$, Vincenzo Parisi', \\ Mariano Serrao ${ }^{4}$, Maurizio Evangelista ${ }^{5}$, Anna Ambrosini ${ }^{6}$, \\ Claudio Colonnese ${ }^{2,6}$, Jean Schoenen ${ }^{7}$ and Francesco Pierelli ${ }^{4,6}$
}

\begin{abstract}
Background: Previous functional MRI studies have revealed that ongoing clinical pain in different chronic pain syndromes is directly correlated to the connectivity strength of the resting default mode network (DMN) with the insula. Here, we investigated seed-based resting state DMN-insula connectivity during acute migraine headaches.

Methods: Thirteen migraine without aura patients (MI) underwent $3 \mathrm{~T}$ MRI scans during the initial six hours of a spontaneous migraine attack, and were compared to a group of 19 healthy volunteers (HV). We evaluated headache intensity with a visual analogue scale and collected seed-based MRI resting state data in the four core regions of the DMN: Medial prefrontal cortex (MPFC), posterior cingulate cortex (PCC), and left and right inferior parietal lobules (IPLs), as well as in bilateral insula.

Results: Compared to HV, MI patients showed stronger functional connectivity between MPFC and PCC, and between MPFC and bilateral insula. During migraine attacks, the strength of MPFC-to-insula connectivity was negatively correlated with pain intensity.

Conclusion: We show that greater subjective intensity of pain during a migraine attack is associated with proportionally weaker DMN-insula connectivity. This is at variance with other chronic extra-cephalic pain disorders where the opposite was found, and may thus be a hallmark of acute migraine head pain.
\end{abstract}

\section{Keywords}

Brain networks, insula, seed-based resting state, magnetic resonance imaging, migraine

Date received: II February 20I7; revised: 19 April 20I7; accepted: 19 May 2017

\section{Introduction}

During the last decades, neuroimaging methods have greatly contributed to the understanding of the central nervous system mechanisms involved in migraine pathophysiology, showing that the disorder is associated with macro- and microstructural, neurochemical, and functional alterations in various brain areas (1). However, despite enormous progress in the field, the brain circuitry subtending and encoding the perception of head pain is still partly elusive and presents several methodological obstacles. Most studies have assessed functional brain changes in response to experimentally induced noxious stimuli or during induced migraine attacks by nitric oxide donors, various other vasodilating chemicals or hypoxia (2-5). PET recordings during
'G.B. Bietti Foundation IRCCS, Research Unit of Neurophysiology of Vision and Neurophthalmology, Rome, Italy

2“'Sapienza” University of Rome, Department of Neurology and Psychiatry, Neuroradiology section, Rome, Italy

${ }^{3}$ Don Carlo Gnocchi Onlus Foundation, Milan, Italy

4"Sapienza" University of Rome Polo Pontino, Department of Medicosurgical Sciences and Biotechnologies, Latina, Italy

${ }^{5}$ Università Cattolica del Sacro Cuore/CIC, Istituto di Anestesiologia, Rianimazione e Terapia del Dolore, Rome, Italy

${ }^{6}$ IRCCS Neuromed, Pozzilli (IS), Italy

${ }^{7}$ Headache Research Unit, Department of Neurology-CHR Citadelle, University of Liège, Belgium

\section{Corresponding author:}

Gianluca Coppola, Research Unit of Neurophysiology of Vision and Neurophthalmology, G.B. Bietti Foundation-IRCCS, Via Livenza 300198 Rome, Italy.

Email: gianluca.coppola@gmail.com 
spontaneous attacks have focused on brain stem and photophobia-related activations (6-10), but the central nervous system underpinnings of headache intensity were not assessed.

Among the various neuroimaging procedures, resting state functional MRI (RS-fMRI) analyses spontaneous BOLD signal fluctuations not attributable to an explicit in- or output, and determines between which brain areas resting activity is related (11). Seed-based or region of interest (ROI) analysis is one method of identifying networks and connectivity in the brain. In this case, the signal from only a certain voxel or a cluster of voxels, known as the seed or ROI, is used to calculate its correlations with other voxels of the brain, which provides a more precise and detailed look at specific connectivity in brain areas of interest. Seed-based analysis of RS-fMRI data is thus suitable for examining the brain activity and connections associated with spontaneous pain, i.e. in the absence of any experimental intervention.

As in certain chronic pain conditions (12-14), seedbased RS-fMRI studies in migraine between attacks have provided evidence of a disruption of the default mode network (DMN) (15-20), a set of brain regions active at rest and involved in self-referential orientation and monitoring (21). Intriguingly, in chronic pain disorders, such as fibromyalgia and low-back pain, such studies have revealed a positive correlation between the intensity of pain and resting DMN connectivity with the insula (INS) $(22,23)$ and this correlation persists with manoeuvre-induced exacerbation of pain in chronic low back pain patients (24). To the best of our knowledge, there are no RS-fMRI studies using seedbased analysis to determine the temporal dynamics of the functional connectivity between DMN and insula during spontaneous migraine attacks, or a fortiori its correlation with headache intensity.

We decided therefore to use a seed-based approach to search for abnormal functional connectivity patterns of the DMN and between the DMN, insula, and the severity of head pain in episodic migraine without aura patients scanned during an attack. We hypothesized that, akin to other pain disorders, migraine headache would be associated with increased connectivity of the DMN with insula and the strength of this connectivity would be proportional to headache intensity.

\section{Materials and methods}

\section{Participants}

Between 2014 and 2016, we performed a comprehensive battery of neuroimaging tests in a large cohort of migraine patients, including RS-fMRI in migraine, for which the results obtained with diffusion tensor imaging (25), voxel-based morphometry (26) and independent component analysis of resting state connectivity (27) are published elsewhere. Thirteen patients who participated in this study were fortuitously scanned during the initial six hours of a spontaneous fullblown migraine attack and included in the present seed-based RS-fMRI analysis. They suffered from migraine without aura (MI, ICHD-3 beta code 1.1), were right-handed and devoid of all the following: A history of other neurological diseases, systemic hypertension, diabetes, or other metabolic disorders, connective or autoimmune diseases, and any other type of primary or secondary headache; fixed unilateral headaches; preventive anti-migraine drugs during the preceding three months; acute anti-migraine drugs before or during the imaging session. We collected clinical data from two-month headache diaries during the screening visit or the recording session (Table 1). Before the fMRI recordings, patients were asked to evaluate their headache intensity on a $10-\mathrm{cm}$ visual analogue scale (VAS). The control group comprised 19 right-handed healthy volunteers (HV) of comparable age and gender distribution. HV had no personal or family history of migraine or epilepsy, or regular medication use. All scanning sessions were performed in the afternoon (4.00-7.00 pm) and females were scanned at mid-cycle. None of the recorded participants were sleep-deprived or consumed alcohol the day preceding the scans. Caffeinated beverages were not allowed on the day of recordings. A further exclusion criterion for both HV and MI was evidence of a brain lesion on structural magnetic resonance imaging.

All participants received a complete description of the study and granted written informed consent. The institutional review board of the Faculty of Pharmacy and Medicine, University of Rome, Polo Pontino, Italy, approved the project. The study complied with the principles set out in the WMA Declaration of

Table I. Clinical and demographic characteristics of healthy volunteers $(\mathrm{HV})$ and migraine without aura patients recorded during (MI) attacks. Data are expressed as means \pm SD.

\begin{tabular}{llc}
\hline & $\begin{array}{l}\mathrm{HV} \\
(\mathrm{n}=19)\end{array}$ & $\begin{array}{l}\mathrm{MI} \\
(\mathrm{n}=13)\end{array}$ \\
\hline Women $(\mathrm{n})$ & 12 & 10 \\
Age (years) & $31.7 \pm 4.0$ & $34.4 \pm 10.7$ \\
$\begin{array}{l}\text { Duration of migraine } \\
\quad \text { history (years) }\end{array}$ & & $15.0 \pm 9.6$ \\
Attack frequency/month $(\mathrm{n})$ & & $3.9 \pm 3.1$ \\
Attack duration (hours) & & $43.4 \pm 28.4$ \\
Visual analogue scale $(\mathrm{n})$ & & $7.4 \pm 0.7$ \\
\hline
\end{tabular}


Helsinki - Ethical principles for medical research involving human subjects.

\section{Imaging protocols}

MRI data were obtained on a Siemens $3 \mathrm{~T}$ Verio scanner using a 12-channel head coil. Structural anatomic scans were performed using a T1-weighted sagittal magnetization-prepared rapid gradient echo (MPRAGE) series (TR: $1900 \mathrm{~ms}$, TE: $2.93 \mathrm{~ms}, 176$ sagittal slices, $0.508 \times 0.508 \times 1 \mathrm{~mm}^{3}$ voxels). We acquired an interleaved double-echo Turbo Spin Echo sequence proton density and T2-weighted images (repetition time: $3320 \mathrm{~ms}$, echo time: $10 / 103 \mathrm{~ms}$, matrix: $384 \times$ 384, field of view: $220 \mathrm{~mm}$, slice thickness: $4 \mathrm{~mm}$, gap: $1.2 \mathrm{~mm}, 50$ axial slices). Functional MRI data were obtained using $\mathrm{T} 2 *$-weighted, echo-planar imaging (TR: $3000 \mathrm{~ms}$, TE: $30 \mathrm{~ms}$, 40 axial slices, $3.906 \times$ $3.906 \times 3 \mathrm{~mm}, 150$ volumes). To accelerate fMRI acquisitions and minimize distortions, the actual EPI uses multiband sequences with simultaneous echo refocusing and parallel imaging (called Generalized Autocalibrating Partially Parallel Acquisition, GRAPPA, by Siemens). Functional resting scans lasted seven minutes and 30 seconds, during which participants were instructed to relax, avoid motion, and keep their eyes closed.

\section{Data processing and analyses}

All images were processed using SPM12 (Statistical Parametric Mapping, version 12). Functional connectivity analyses were performed using the CONN v16b connectivity toolbox (28) in MATLAB (MathWorks, Inc).

Pre-processing involved the following steps based on SPM12 algorithms. All images from a single subject were realigned using a six-parameter rigid body process, resliced by a cubic spline interpolation; outcomes, motion, and rotations were recorded in a text file for every subject and used as first level covariates. The structural (T1 - MPRAGE) and functional data were co-registered for each subject dataset; data were transformed into a common stereotactic space based on Talairach and Tournoux (29) and resampled isotropically at $3 \times 3 \times 3 \mathrm{~mm}$. After that, the spatially normalized functional images were smoothed by $8 \mathrm{~mm}$ in each direction.

The processing steps listed below were performed using CONN, following the procedure reported elsewhere (30). First, each participant's structural dataset was segmented to grey matter, white matter, and cerebrospinal fluid (CSF). The pre-process step removes sources of possible confounders: BOLD signals from white matter and CSF, realignment parameters of each subject as covariates. Moreover, a scrubbing preprocessing step based on the CONN artefact detector tool (ART) detected the offending scans for every subject; therefore the output text files were used in the denoising step for scrubbing. None of the participants' volumes was removed from the analysis due to excessive motion. The rest effect condition convolved with hemodynamic response function and the bandpass filter values were 0.01 and $0.1 \mathrm{~Hz}$.

First level functional connectivity analysis was performed using the seed-based approach. We collected seed-based resting state data in the four core regions of the DMN: Medial prefrontal cortex (MPFC), posterior cingulate cortex (PCC), and bilateral inferior parietal lobules (IPLs). In addition, we collected seed-based resting state data from the insula bilaterally. All seeds - or region of interests, ROIs - were independent from our data and derived from the Automated Anatomical Labeling (AAL) atlas (31). A total of six ROIs were defined. Thereafter, functional connectivity of the different seeds was determined in each single subject, based on a bivariate correlation method.

The images resulting from the first level analysis provided the seed-to-voxel connectivity maps for each source, for each subject and for the rest condition. We used these data to compute the Pearson's correlation maximum coefficient value between the time course of each of the four DMN areas and that of bilateral insula for each subject.

The second level connectivity analysis used a general linear model analysis to investigate within-group and between-group (HV vs. MI) combinations of effects, using the DMN areas and insulae as contrasts and the subjects as regressors.

We also calculated $t$-scores (uncorrected $p$-values) between each pair of seed areas (ROI-to-ROI analysis).

\section{Statistical analysis}

Group differences for demographic data were assessed using ANOVA. To explore the within-group connectivity patterns, a one-sided one sample $t$-test was performed on the individual normalized connectivity maps in each group (HV and MI).

The DMN functional connectivity maps in each group were obtained using the independent component analysis (ICA) analysis toolbox in GIFT v4.0 (32) without correction at a significance level of 0.001 and a minimum cluster size of five voxels. These functional connectivity maps were used to visually represent the spatial characteristics of the DMN areas in Figure 1.

The difference between groups of connectivity between the four DMN areas and bilateral insula was evaluated using an unpaired two sample $t$-test with an uncorrected threshold of $p<0.05$.

We used Pearson's test to search for correlations between each of the four DMN ROIs and the insula 


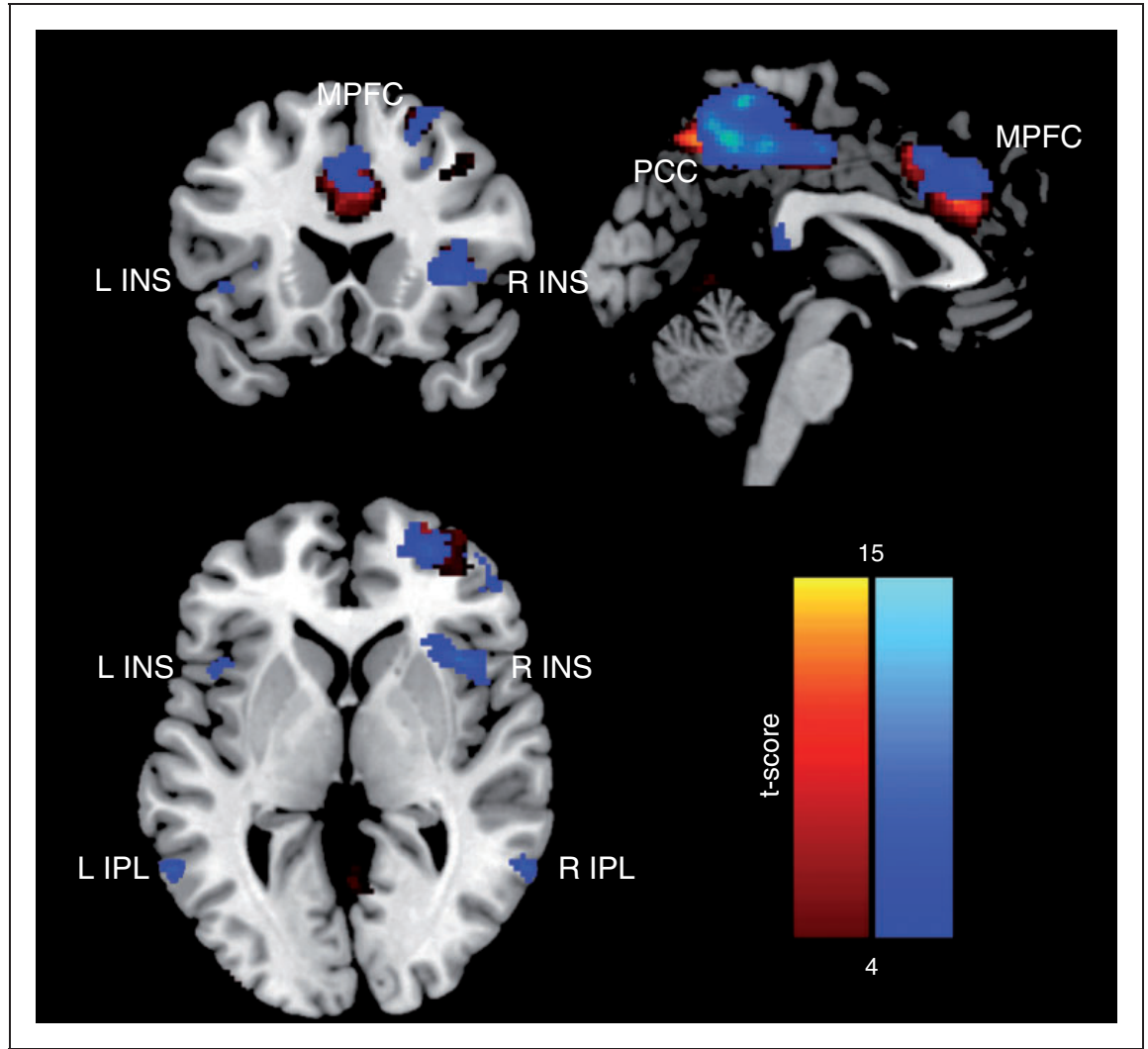

Figure I. Spatial characteristics of the areas of the Default Mode Network included in the seed-based resting state analysis for MI patients (in blue) and HV (in yellow/red): Posterior cingulate cortex (PCC), inferior parietal lobule (IPL), medial prefrontal cortex (MPFC). We also collected seed-based resting state data from the insula (INS) bilaterally.

bilaterally in each subject, and to relate these values (separately for right and left insula) to individual VAS scores, as well as other clinical variables. Correlations with a $p$ value $\leq 0.05$ were considered significant.

\section{Results}

All 32 subjects completed the scanning sessions. Demographic variables are summarized in Table 1. Patients reported no premonitory symptoms prior to the headache phase. Mean headache intensity immediately before the scans was $7.7 \pm 0.9$ (range: 6-10) on the $10 \mathrm{~cm}$ visual analogue scale. None of the patients withdrew from the study due to migraine symptoms or to MRI-induced discomfort. Structural brain MRIs were normal in all participants.

\section{Seed-based resting state $\mathrm{FMRI}$}

To visualize the strength of the DMN connectivity, we created group-level t-maps for healthy volunteers (HV) and ictal migraine patients (MI).

The two sample $t$-test disclosed a significantly greater correlation in MI between MPFC and PCC $(t=2.33, p=0.013)$ than in HV (Figure 1). MI patients also exhibited stronger connectivity than HV between MPFC or right IPL and right $(t=1.71, p=0.049$; $t=1.82, p=0.039$, respectively) or left insula $(t=1.91$, $p=0.033 ; t=1.76, p=0.044$, respectively) (Figure 2). We failed to find a significant correlation between PCC and insula bilaterally.

\section{Clinical correlations}

In MI, the strength of MPFC-to-INS connectivity was negatively correlated with subjective perception of migraine headache severity as measured by the VAS score (right $\mathrm{F}=5.59, \quad p=0.038, \quad \mathrm{r}=-0.585$, $\mathrm{R}$-sq(adj) $=27.7 \%$; left $\mathrm{F}=5.43, p=0.040 \mathrm{r}=-0.575$, $R-s q(\operatorname{adj})=26.95 \%$ ) (Figure 3$)$. We found no significant correlation between other clinical features of migraine and MPFC-to-INS connectivity or any other ROI-to-ROI connectivity.

\section{Discussion}

The main findings of our study can be summarized as follows: 1) compared with $\mathrm{HV}$, migraine patients had stronger intrinsic connectivity between MPFC and PCC regions of the DMN; 2) in $\mathrm{MI}$, seed-based 


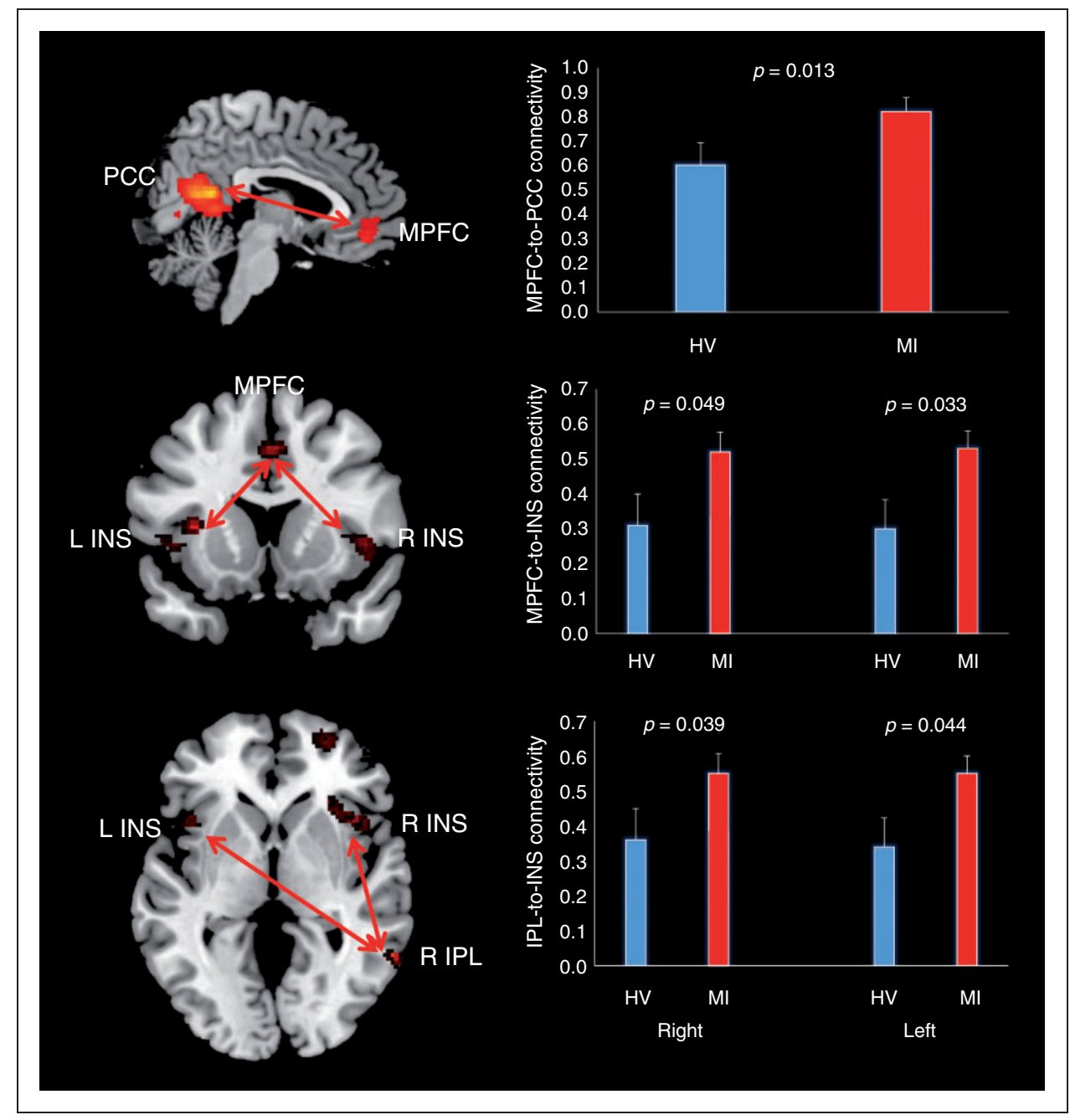

Figure 2. Default Mode Network (DMN) inter-regional resting state-functional connectivity in migraine patients during an attack (MI) and healthy volunteers (HV). Left panel: Illustrative image from an Ml patient showing activated DMN areas overlaid on the "ch2better" brain template image for visualization. Right panel: Histograms of correlation coefficients ( $r$ ) between middle prefrontal cortex (MPFC) and posterior cingulate cortex (PCC) (upper), between MPFC and right and left insula (INS) (middle), and between right inferior parietal lobule (IPL) and right and left INS (lower). Correlations (mean + standard errors) are significantly greater in MI than in HV for all connectivities.

analyses disclosed increased connectivity of MPFC and right IPL DMN areas to bilateral insula; and 3) contrary to our initial hypothesis, the strength of MPFC-to-insula connectivity was negatively correlated with the severity of perceived migraine headache. Altogether, our findings identify the insula bilaterally and MPFC as key areas of maladaptive intrinsic connectivity during migraine attacks.

\section{Interregional DMN connectivity}

To the best of our knowledge, abnormal connectivity between areas of DMN integrity have not been reported before during migraine attacks. Disruption of intra-DMN connectivity was, however, found in migraine patients who were scanned between attacks
(15-20). Interictal spontaneous activity was significantly decreased in the major regions of the DMN (15-18), including the PCC, IPL and MPFC, and the latter activity was negatively correlated with the duration of the migraine disorder $(16,17)$. In an independent component RS-fMRI analysis we recently found, in migraine between attacks, that the DMN was less connected with a network composed of visuo-spatial and medial visual cortical areas, and that the strength of the latter network was in turn associated with microstructural thalamic abnormalities (19). Here, with a seed-based analysis, migraine patients scanned during an attack had a significant increase in connectivity strength between MPFC and PCC, ROIs belonging to the DMN, as compared with HV, instead of a decrease as previously reported interictally. 


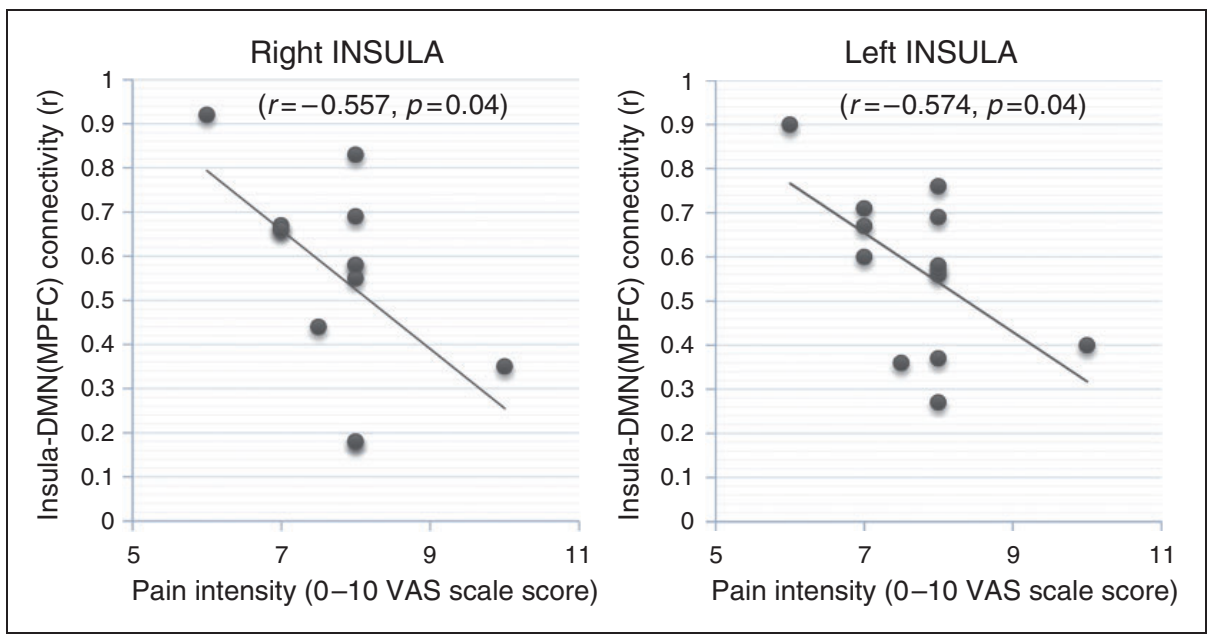

Figure 3. In patients, MPFC-to-insula connectivity is negatively correlated with the intensity of the migraine headache as measured on a visual analogue scale.

Among the set of DMN regions, the midline MPFC and of PCC have distinct roles $(21,33)$. The PCC is a tonically active brain region that may continuously gather extrinsic, and possibly intrinsic information (21). The PCC responds to both emotional and nonemotional stimuli (34). A large body of data assigns to the MPFC a role in the integrative processing of emotional and cognitive processes by incorporating emotional biasing signals or markers into decisionmaking processes (21). It appears that MPFC and PCC normally act synergistically. In fact, Kucyi et al. (35) showed that when healthy subjects' minds wander away from incoming nociceptive stimulations, there is increased connectivity between the MPFC and PCC regions of the $\mathrm{DMN}$, as well as areas belonging to the salience network (SN) such as the insula. Concordantly, we show that, in patients who are at rest during the headache phase, connectivity between PCC and MPFC is increased, and that MPFC is highly connected with bilateral insula. This might be the consequence of a spontaneous tendency of some migraineurs to try to mind-wander away from their pain because of the absence of other immediately available coping strategies.

\section{DMN-to-insula connectivity}

In animal studies, the insular cortex, anchored with the $\mathrm{SN}$, is reciprocally connected to multiple sensory, motor, limbic, and association areas of the brain (36). The insular cortex and areas within the DMN share significant topographic reciprocal connectivity and form an anatomically tightly coupled network ideally placed to integrate information from several brain regions (36). This emphasizes that the insula has numerous functions; for instance, it takes part in processing subjective emotional experiences, in sensorimotor integration, in goal-directed cognition, and in autonomic response. Moreover, the insula may channel inputs into the $\mathrm{SN}$ and regulate spontaneous switching between the DMN and the executive control network (ECN) to optimize performance (37). Previous neuroimaging studies during migraine attacks have repeatedly provided evidence of activation in bilateral insula, which was considered as a non-specific response to pain $(2,4,9,10,38)$. Between attacks, migraineurs also had abnormal insular cortex connectivity $(39,40)$ and habituation in response to repeated sensory stimuli $(41,42)$. In the present study, we show that migraine patients during an attack have greater bilateral insula connectivity with MPFC and right IPL areas of the DMN than healthy volunteers, in the absence of a specific task. Taken together with our previous results obtained in the same group of subjects showing reduced functional connectivity between the ECN and the attentive networks (27), the present findings suggest that, during a migraine attack, the control by the insula of the spontaneous switching between the DMN and the ECN to optimize performance is abnormal, favouring activation of the former over the latter. Additionally, since the prefrontal cortex is deemed to mediate attenuation of pain via cognitive control mechanisms (43), increased MPFC-to-insula connectivity during the migraine attack could be a physiological mechanism to cognitively attenuate pain perception.

\section{DMN-to-INS correlation with severity of migraine headache}

Another striking finding in our study is the correlation between DMN-to-insula connectivity and severity of the migraine headache. During migraine attacks, individual r-scores of MPFC-to-bilateral insula 
connectivity correlated negatively with subjective perception of headache intensity. Remarkably, this finding is at variance with previous studies on the association between clinical pain and DMN connectivity in chronic pain disorders like fibromyalgia and chronic low back pain. As a matter of fact, in these studies greater clinical pain was associated with greater MPFC connectivity with the insula (22-24) and not lesser, as shown here. This raises the intriguing possibility that the anti-correlation between MPFC-to-insula connectivity and head pain severity is a distinct feature of episodic migraine attacks. It may also be related to the finding, in patients between attacks, that connectivity between insula and the DMN is positively correlated with the usual duration of migraine attacks (15). Moreover, when taken separately, the regional metabolism of the insular cortex (44) and the regional homogeneity within the MPFC (17) are significantly anti-correlated with disease duration, and insular metabolism is anti-correlated with lifetime headache frequency (44). Further studies are needed to verify whether the aberrant ictal/interictal connectivity patterns in migraine are permanent functional abnormalities of a progressive brain disorder or, more likely, plastic changes of brain functions relative to the time of an attack, as previously clearly shown with clinical neurophysiology methods (45).

Interestingly, besides pain-induced DMN and insula activation, the above-mentioned study by Kucyi et al. (35) has shown stronger dynamic functional connectivity between MPFC and periaqueductal gray (PAG), an opiate-rich region mediating pain suppression, suggesting that sustained DMN activity during pain, for instance during head pain as in our study, may be a physiological strategy of the brain to try to reduce or limit pain (46). In this context, further studies are worthwhile to determine whether the microstructural changes in the PAG and its abnormal connectivity with the prefrontal cortex $(47,48)$, particularly with worsening of attack frequency (49) and intensity (50), may be primary dysfunctions or secondary maladaptive consequences of the abnormal connectivity between networks during the migraine cycle.

It also remains to be determined if the abnormal DMN-to-INS connectivity could be a consequence of a 'thalamocortical dysrhythmia', a model for the cyclic functional brain abnormalities found in migraine $(51,52)$. For instance, Wang et al. (53) found that inflow/outflow signals between the posterior thalamic nuclei and the limbic system, MPFC, and temporo-parieto-occipital areas were decreased in relation to the severity of migraine clinical features. This might support the hypothesis that a disrupted thalamic control of brain networks, such as that found by us previously in patients during spontaneous attacks (27), could underlie the distinct resting state connectivity patterns found in the present study.

We acknowledge some limitation of our study. Firstly, cross-sectional fMRI studies limit inferences about causality. Secondly, as all patients were ictal at the time of scanning, the results provide no information about brain functions between attacks. It is nevertheless striking that disruptions of the DMN and insula connectivity have been consistently observed in episodic migraine also between attacks. Future work using the same methods is necessary to follow patients longitudinally, i.e. during attacks as well as at different time points during the pain-free periods. Since our study sample is rather small, larger studies are also needed to replicate our findings and to include highfrequency and chronic migraine patients.

\section{Conclusions}

In patients recorded during a full-blown migraine attack, we report increased connectivity between areas belonging to the DMN as well between the latter and bilateral insula. Moreover, we show that the strength of the DMN-to-INS connectivity is anticorrelated with intensity of the migraine headache, which contrasts with the opposite finding in chronic pain disorders. Altogether, these results support, on the one hand, resting DMN-to-INS connectivity as a state-specific neuroimaging marker for the encoding of the clinical characteristics of pain, and, on the other hand, significantly extend these data by providing evidence that a distinct connectivity pattern may encode intensity of acute migraine headache. Hence, comparative studies of other acute painful conditions are necessary to verify the specificity of these findings for migraine.

\section{Article highlights}

- How the brain encodes the severity of acute migraine headache is unknown.

- We found that migraineurs during attacks had stronger intrinsic default-mode network (DMN) connectivity and that the strength of DMN-to-insula connectivity was anticorrelated with the severity of headache, which is at variance with publications on chronic pain disorders. 


\section{Acknowledgment}

The contribution of the G.B. Bietti Foundation in this paper was supported by the Ministry of Health and Fondazione Roma.

\section{Declaration of conflicting interests}

The authors declared no potential conflicts of interest with respect to the research, authorship, and/or publication of this article.

\section{Funding}

The authors disclosed receipt of the following financial support for the research, authorship, and/or publication of this article: Fondazione Roma, Italian Ministry of Health.

\section{References}

1. Sprenger T and Borsook D. Migraine changes the brain: Neuroimaging makes its mark. Curr Opin Neurol 2012; 25: 252-262.

2. Bahra A, Matharu MS, Buchel C, et al. Brainstem activation specific to migraine headache. Lancet 2001; 357: 1016-1017.

3. Hansen JM, Hauge AW, Olesen J, et al. Calcitonin gene-related peptide triggers migraine-like attacks in patients with migraine with aura. Cephalalgia 2010; 30: 1179-86.

4. Amin FM, Hougaard A, Magon S, et al. Change in brain network connectivity during PACAP38-induced migraine attacks: A resting-state functional MRI study. Neurology 2016; 86: 180-187.

5. Arngrim N, Schytz HW, Britze J, et al. Migraine induced by hypoxia: an MRI spectroscopy and angiography study. Brain 2016; 139: 723-737.

6. Denuelle M, Boulloche N, Payoux P, et al. A PET study of photophobia during spontaneous migraine attacks. Neurology 2011; 76: 213-218.

7. Weiller C, May A, Limmroth V, et al. Brain stem activation in spontaneous human migraine attacks. Nat Med 1995; 1: 658-660.

8. Bednarczyk EM, Remler B, Weikart C, et al. Global cerebral blood flow, blood volume, and oxygen metabolism in patients with migraine headache. Neurology 1998; 50: 1736-1740.

9. Afridi SK, Giffin NJ, Kaube H, et al. A positron emission tomographic study in spontaneous migraine. Arch Neurol 2005; 62: 1270-1275.

10. Stankewitz A and May A. Increased limbic and brainstem activity during migraine attacks following olfactory stimulation. Neurology 2011; 77: 476-482.

11. Fox $M$ and Raichle ME. Spontaneous fluctuations in brain activity observed with functional magnetic resonance imaging. Nat Rev 2007; 8: 700-711.

12. Cauda F, Sacco K, Duca S, et al. Altered resting state in diabetic neuropathic pain. PLoS One 2009; 4: e4542.

13. Baliki MN, Baria AT and Apkarian AV. The cortical rhythms of chronic back pain. J Neurosci 2011; 31: 13981-13990.
14. Cifre I, Sitges C, Fraiman D, et al. Disrupted functional connectivity of the pain network in fibromyalgia. Psychosom Med 2012; 74: 55-62.

15. Xue T, Yuan K, Zhao L, et al. Intrinsic brain network abnormalities in migraines without aura revealed in resting-state fMRI. PLoS One 2012; 7: 10.

16. Xue T, Yuan K, Cheng P, et al. Alterations of regional spontaneous neuronal activity and corresponding brain circuit changes during resting state in migraine without aura. NMR Biomed 2013; 26: 1051-1058.

17. Yu D, Yuan K, Zhao L, et al. Regional homogeneity abnormalities in patients with interictal migraine without aura: A resting-state study. NMR Biomed 2012; 25 : 806-812.

18. Tessitore A, Russo A, Giordano A, et al. Disrupted default mode network connectivity in migraine without aura. J Headache Pain 2013; 14: 89.

19. Coppola G, Di Renzo A, Tinelli E, et al. Thalamo-cortical network activity between migraine attacks: Insights from MRI-based microstructural and functional restingstate network correlation analysis. J Headache Pain 2016; 17: 100 .

20. Zhang J, Su J, Wang M, et al. Increased default mode network connectivity and increased regional homogeneity in migraineurs without aura. J Headache Pain 2016; 17 : 98.

21. Raichle M, MacLeod AM, Snyder AZ, et al. A default mode of brain function. Proc Natl Acad Sci USA 2001; 98: 676-682.

22. Napadow V, LaCount L, Park K, et al. Intrinsic brain connectivity in fibromyalgia is associated with chronic pain intensity. Arthritis Rheum 2010; 62: 2545-2555.

23. Napadow V, Kim J, Clauw DJ, et al. Decreased intrinsic brain connectivity is associated with reduced clinical pain in fibromyalgia. Arthritis Rheum 2012; 64: 2398-2403.

24. Loggia ML, Kim J, Gollub RL, et al. Default mode network connectivity encodes clinical pain: An arterial spin labeling study. Pain 2013; 154: 24-33.

25. Coppola G, Tinelli E, Lepre C, et al. Dynamic changes in thalamic microstructure of migraine without aura patients: A diffusion tensor magnetic resonance imaging study. Eur J Neurol 2014; 21: 287-e13.

26. Coppola G, Di Renzo A, Tinelli E, et al. Evidence for brain morphometric changes during the migraine cycle: A magnetic resonance-based morphometry study. Cephalalgia 2015; 35: 783-791.

27. Coppola G, Di Renzo A, Tinelli E, et al. Thalamo-cortical network activity during spontaneous migraine attacks. Neurology 2016; 87: 2154-2160.

28. CONN: Functional connectivity toolbox, https://www. nitrc.org/projects/conn (2016, accessed 11 February 2017).

29. Talairach J and Tournoux P. Co-planar Stereotaxic Atlas of the Human Brain 1998. Stuttgart, Germany: Thieme.

30. Whitfield-Gabrieli S and Nieto-Castanon A. Conn: A functional connectivity toolbox for correlated and anticorrelated brain networks. Brain Connect 2012; 2: 125-141.

31. Tzourio-Mazoyer N, Landeau B, Papathanassiou D, et al. Automated anatomical labeling of activations in 
SPM using a macroscopic anatomical parcellation of the MNI MRI single-subject brain. Neuroimage 2002; 15: 273-289.

32. GIFT v4.0, http://mialab.mrn.org/software/gift/index. html (2017, accessed 18 April 2017).

33. Buckner RL, Andrews-Hanna JR and Schacter DL. The brain's default network: Anatomy, function, and relevance to disease. Ann N Y Acad Sci 2008; 1124: 1-38.

34. Vogt BA, Vogt L and Laureys S. Cytology and functionally correlated circuits of human posterior cingulate areas. Neuroimage 2006; 29: 452-466.

35. Kucyi A, Salomons TV and Davis KD. Mind wandering away from pain dynamically engages antinociceptive and default mode brain networks. Proc Natl Acad Sci USA 2013; 110: 18692-18697.

36. Mesulam MM and Mufson EJ. Insula of the old world monkey. III: Efferent cortical output and comments on function. J Comp Neurol 1982; 212: 38-52.

37. Sridharan D, Levitin DJ and Menon V. A critical role for the right fronto-insular cortex in switching between central-executive and default-mode networks. Proc Natl Acad Sci 2008; 105: 12569-12574.

38. Denuelle M, Fabre N, Payoux P, et al. Hypothalamic activation in spontaneous migraine attacks. Headache 2007; 47: 1418-1426.

39. Hadjikhani N, Ward N, Boshyan J, et al. The missing link: Enhanced functional connectivity between amygdala and visceroceptive cortex in migraine. Cephalalgia 2013; 33: 1264-1268.

40. Mathur VA, Moayedi M, Keaser ML, et al. High frequency migraine is associated with lower acute pain sensitivity and abnormal insula activity related to migraine pain intensity, attack frequency, and pain catastrophizing. Front Hum Neurosci 2016; 10: 489.

41. Stankewitz A, Schulz E and May A. Neuronal correlates of impaired habituation in response to repeated trigemino-nociceptive but not to olfactory input in migraineurs: An fMRI study. Cephalalgia 2013; 33: 256-265.

42. Lee J, Lin RL, Garcia RG, et al. Reduced insula habituation associated with amplification of trigeminal brainstem input in migraine. Cephalalgia. Epub ahead of print 13 August 2016. DOI: 10.1177/ 0333102416665223.

43. Wiech K, Ploner M and Tracey I. Neurocognitive aspects of pain perception. Trends Cogn Sci 2008; 12: 306-313.

44. Kim JH, Kim S, Suh S-I, et al. Interictal metabolic changes in episodic migraine: A voxel-based FDG-PET study. Cephalalgia 2010; 30: 53-61.

45. Coppola G, Di Lorenzo C, Schoenen J, et al. Habituation and sensitization in primary headaches. $J$ Headache Pain 2013; 14: 65.

46. Welberg L. Pain: A wandering brain reduces pain? Nat Rev Neurosci 2013; 14: 819.

47. DaSilva AFM, Granziera C, Tuch DS, et al. Interictal alterations of the trigeminal somatosensory pathway and periaqueductal gray matter in migraine. Neuroreport 2007; 18: 301-305.

48. Ito K, Kudo M, Sasaki M, et al. Detection of changes in the periaqueductal gray matter of patients with episodic migraine using quantitative diffusion kurtosis imaging: Preliminary findings. Neuroradiology 2016; 58: 115-120.

49. Mainero C, Boshyan J and Hadjikhani N. Altered functional magnetic resonance imaging resting-state connectivity in periaqueductal gray networks in migraine. Ann Neurol 2011; 70: 838-845.

50. Li Z, Liu M, Lan L, et al. Altered periaqueductal gray resting state functional connectivity in migraine and the modulation effect of treatment. Sci Rep 2016; 6: 20298.

51. Llinás RR and Steriade M. Bursting of thalamic neurons and states of vigilance. J Neurophysiol 2006; 95: 3297-3308.

52. Coppola G, Ambrosini A, Clemente L Di, et al. Interictal abnormalities of gamma band activity in visual evoked responses in migraine: An indication of thalamocortical dysrhythmia? Cephalalgia 2007; 27: 1360-1367.

53. Wang T, Zhan W, Chen Q, et al. Altered restingstate ascending/descending pathways associated with the posterior thalamus in migraine without aura. Neuroreport 2016; 27: 257-263. 